\title{
Extrusão de Compósitos de PP com Fibras Curtas de Coco: Efeito da Temperatura e Agentes de Acoplamento
}

\author{
Evelise F. Santos, Mauro Moresco, Simone M. L. Rosa, Sônia M. B. Nachtigall \\ Instituto de Química, UFRGS
}

\begin{abstract}
Resumo: Compósitos de PP com fibras curtas de coco foram preparados em extrusora, com dois perfis de temperatura, visando avaliar o efeito compatibilizante de PP modificado com vinilalcoxisilano e com anidrido maleico. Foi verificado que os dois agentes de acoplamento melhoraram o módulo elástico, a tensão máxima e a resistência à absorção de água quando os materiais foram processados utilizando-se um perfil de temperaturas mais elevadas. A morfologia dos compósitos também ficou mais homogênea na presença dos agentes de acoplamento, especialmente naqueles processados na temperatura mais alta. Esses resultados indicam que a temperatura é uma variável fundamental no estabelecimento das interações envolvendo os processos de compatibilização.
\end{abstract}

Palavras-chave: Compósitos, fibras naturais, agentes de acoplamento, extrusão, absorção de água.

\section{Extrusion of PP Composites with Short Coir Fibers: Effect of Temperature and Coupling Agents}

Abstract: PP composites filled with coir short fibers were prepared in an extruder using two temperature profiles. The objective was to evaluate the compatibilizing effect of PP modified with vinylsilane and with maleic anhydride. Both coupling agents improved elastic modulus, tensile strength and water absorption resistance when the materials were processed at the highest temperature profile. The composite morphology was more homogeneous in the presence of the coupling agents, mainly in the composites processed at the highest temperatures. These results indicate that temperature is a key variable for the establishment of the interactions involved in the coupling processes.

Keywords: Composites, natural fibers, coupling agent, extrusion, silane.

\section{Introdução}

O desenvolvimento sustentável, a redução da demanda por materiais de origem fóssil e a redução da produção de resíduos são fatores que se relacionam entre si e que, bem conduzidos, podem resultar em benefícios importantes para a vida humana ${ }^{[1]}$. Nesse contexto o Brasil, na qualidade de concentrador de uma das mais ricas biodiversidades do planeta, tem um papel fundamental ${ }^{[2]}$. O crescente interesse no uso de fibras naturais na preparação de compósitos poliméricos constitui uma alternativa de obtenção de novos e interessantes materiais com menor impacto ambiental porque permite substituir parte dos polímeros obtidos a partir de material fóssil por fibras de origem agrícola, de caráter renovável e biodegradável. Além disso, muitas fibras e cargas vegetais constituem resíduos da atividade agrícola e seu aproveitamento, além de lhes prover um destino final racionalizado, pode reduzir os custos de preparação do produto final. Uma das principais aplicações dos compósitos poliméricos com fibras vegetais tem sido na indústria automobilística ${ }^{[3,4]}$, porém seu uso também tem se mostrado promissor na construção civil e na produção de artefatos de uso geral, tais como solados de calçados, pentes, utensílios domésticos e outros.

O Brasil é um país que se destaca na produção de muitas fibras, tais como sisal, rami, algodão, coco e juta, porém diversas outras espécies são encontradas e cultivadas no paíi ${ }^{[5,6]}$. No Brasil, as fibras obtidas da casca do coco são materiais lignocelulósicos de baixo custo, que não encontram aplicação em fins alimentícios, por isso representam uma interessante matéria-prima para a preparação de compósitos poliméricos ${ }^{[7]}$, sendo já empregadas comercialmente na forma de compósitos com elastômeros na produção de assentos de automóveis ${ }^{[8]}$.

O uso de poliolefinas como matrizes na fabricação de compósitos com fibras naturais é limitada pela baixa adesão entre os componentes. A modificação da superfície das fibras e o emprego de agentes de acoplamento têm sido métodos utilizados para aumentar essa adesão ${ }^{[9]}$. O tratamento químico superficial das fibras implica no seu manuseio prévio, com o uso frequente de solventes e posteriores tratamentos térmicos, os quais são desnecessários com a adição de agentes de acoplamento, simplificando assim o processo de fabricação dos compósitos. PP modificado com anidrido maleico (PPAM) tem sido o agente de acoplamento mais utilizado em compósitos de PP com materiais lignocelulósicos ${ }^{[10-12]}$. O mecanismo de compatibilização usualmente descrito propõe a formação de ligações covalentes entre os grupos anidrido reativos do polímero funcionalizado e os grupos hidroxila das cadeias de celulose presentes na fase dispersa $^{[13]}$. Além das ligações covalentes, interações do tipo ligações de hidrogênio também são formadas, conforme ilustrado na Figura 1. O entrelaçamento das cadeias da matriz polimérica com a cadeia carbônica do agente de acoplamento estabelece a interação necessária para promover a adesão entre as fases.

Alcoxisilanos têm se mostrado muito eficientes como agentes de acoplamento em sistemas orgânico-inorgânicos, tais como PP e fibras de vidro $^{[14]}$. Mais recentemente seu uso tem sido estendido para compósitos de polímeros com cargas lignocelulósicas. O mecanismo usualmente proposto para a compatibilização sugere a formação inicial de grupos silanóis, os quais reagem com as hidroxilas da celulose eliminando água ${ }^{[15]}$. Estudo recente, entretanto, mostrou que a hidrólise do silano produzindo grupos silanóis não é condição indispensável para que se estabeleça a adsorção dos silanos nas fibras celulósicas ${ }^{[16]}$, a qual pode ser conseguida também através de interações do tipo van der Waals.

Mondragon e seu grupo ${ }^{[17]}$ compararam o efeito do tratamento de fibras de linho e do tratamento da matriz de PP em compósitos desses materiais, utilizando como agentes de modificação anidrido maleico e viniltrimetoxisilano. Os autores verificaram que o tratamento das fibras não resultou nenhum efeito nas propriedades

Autor para correspondência: Sônia M. B. Nachtigall, Instituto de Química, Universidade Federal do Rio Grande do Sul, Av. Bento Gonçalves, 9500, 
mecânicas dos compósitos, porém a modificação da matriz com esses agentes de acoplamento, na presença de peróxido, produziu um aumento significativo na resistência à tensão e no módulo elástico dos produtos obtidos. Assim, nosso grupo tem proposto a utilização de PP modificado com organosilano como agente de acoplamento em compósitos de PP com diferentes cargas, especialmente cargas vegetais $^{[18-20]}$. Esses estudos mostraram que o PP modificado com viniltrietoxisilano (PPVTES) foi mais eficiente que o PPAM na melhoria das propriedades mecânicas e na redução do grau de absorção de água de compósitos de PP com farinha de madeira preparados em câmara de mistura ${ }^{[19]}$. O mecanismo de acoplamento é semelhante ao proposto para o PPAM, com os grupos alcoxisilanos presentes na matriz funcionalizada reagindo com as hidroxilas presentes na superfície das fibras (Figura 2).

A preparação de compósitos utilizando fibras curtas permite que o processamento seja realizado por extrusão e injeção, métodos geralmente utilizados na indústria para processar matrizes poliméricas. As condições de processamento escolhidas são muito importantes na qualidade da dispersão e na prevenção da aglomeração das fibras na matriz polimérica, especialmente nos compósitos com carga lignocelulósica, pois essas podem ser empregadas em altas concentrações, pelo fato de apresentarem baixo caráter abrasivo ${ }^{[21]}$. Fibras curtas também reduzem os problemas relacionados à dificuldade de alimentação e à ação cisalhante do equipamento, pois quanto menor é o comprimento das fibras, menores são os efeitos da redução de tamanho causados por sua quebra durante o processamento $^{[22,23]}$.

A avaliação da adesão interfacial também é mais fácil de ser feita nos compósitos com fibras curtas já que efeitos de reforço atribuídos ao comprimento das fibras não estão envolvidos ${ }^{[2]}$. Considerando que o processo de extrusão dos compósitos permite obter uma eficiente dispersão das fibras na matriz polimérica é preciso, ainda, garantir que as condições de processamento também favoreçam a atuação dos agentes de acoplamento adicionados para compatibilizar os sistemas. Parâmetros como o tempo de residência

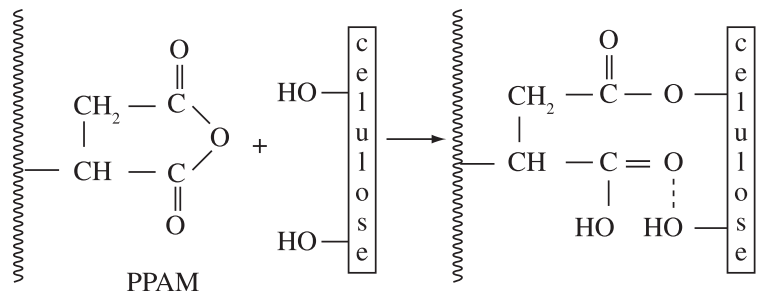

Figura 1. Mecanismo de acoplamento do PPAM com as fibras celulósicas.<smiles>CC(C)C</smiles><smiles>CCO[Si](CC)(OCC)OCC</smiles>

$$
3 \mathrm{H}_{2} \mathrm{O}
$$

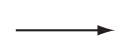
$\mathrm{OCH}_{2} \mathrm{CH}_{3}$<smiles>C[C@@H]1CC[Si](O)(O)O1</smiles><smiles>OC1CCCCC1</smiles>

Figura 2. Mecanismo de acoplamento do PPVTES com as fibras celulósicas. do material na extrusora e o perfil de temperatura utilizado, que podem ser insuficientes para permitir a efetiva ação dos agentes na interface, devem ser avaliados cuidadosamente, visando a obtenção das melhores propriedades. No caso específico de fibras celulósicas um aspecto importante que deve ser considerado é o seu alto caráter hidrofílico, pois a absorção de umidade, além de alterar as dimensões do material devido ao inchamento, pode reduzir significativamente suas propriedades mecânicas.

Neste trabalho foram preparados compósitos de PP com fibras de coco maduro (FC) para a obtenção de um material de baixo custo e com propriedades mecânicas satisfatórias. Para promover a adesão entre as fases foram utilizados dois agentes de acoplamentos macromoleculares: um PP modificado com viniltrietoxisilano (PPVTES) preparado em laboratório e um PP modificado com anidrido maleico (PPAM) comercial. Os compósitos foram preparados em extrusora, utilizando dois diferentes perfis de temperatura, visando comparar a atuação do PPVTES e do PPAM na interface dos compósitos, bem como a influência da temperatura de processamento nas propriedades finais dos materiais. Os compósitos foram caracterizados por testes de tração, Microscopia Eletrônica de Varredura e testes de absorção de água.

\section{Parte Experimental}

\section{Materiais}

PP altamente isotático, densidade $=0,905 \mathrm{~g} \cdot \mathrm{cm}^{-3}, \mathrm{IF}=3,5 \mathrm{~g} / 10 \mathrm{~min}$ $\left(2,16 \mathrm{~kg}, 230{ }^{\circ} \mathrm{C}\right)$, produzido pela BRASKEM SA (Triunfo, Brasil), foi utilizado como matriz polimérica. Fibras de coco fornecidas pela AMAFIBRA (Belém, Brasil) foram utilizadas na forma de fibras curtas (0,5-4 mm). As fibras foram moídas em um moinho de facas e posteriormente passaram por uma classificação granulométrica com a utilização de peneiras. Antes da preparação dos compósitos as fibras foram secas em estufa, com pressão de $\sim 30 \mathrm{~mm} \cdot \mathrm{Hg}^{-1}$, a $80{ }^{\circ} \mathrm{C}$, por 8 horas. PPVTES (IF de $33 \mathrm{~g} / 10 \mathrm{~min}-2,16 \mathrm{~kg}, 230^{\circ} \mathrm{C}$ ) foi preparado conforme descrito na literatura ${ }^{[24]}$. O grau de funcionalização obtido foi 2,4 g\%. PPAM comercial (Orevac CA 100, Atofina), IF > 100 g/ 10 min $\left(2,16 \mathrm{~kg}, 230{ }^{\circ} \mathrm{C}\right)$, apresentando grau de funcionalização $1,0 \mathrm{~g} \%$, foi utilizado como agente de acoplamento.

\section{Processamento e moldagem}

Os compósitos foram processados em uma extrusora duplarosca cônica corrotatória Haake, modelo Rheomex CTW100p, com a velocidade da rosca ajustada em $40 \mathrm{rpm}$. As fibras, o PP e os agentes de acoplamento foram misturados e introduzidos juntos na

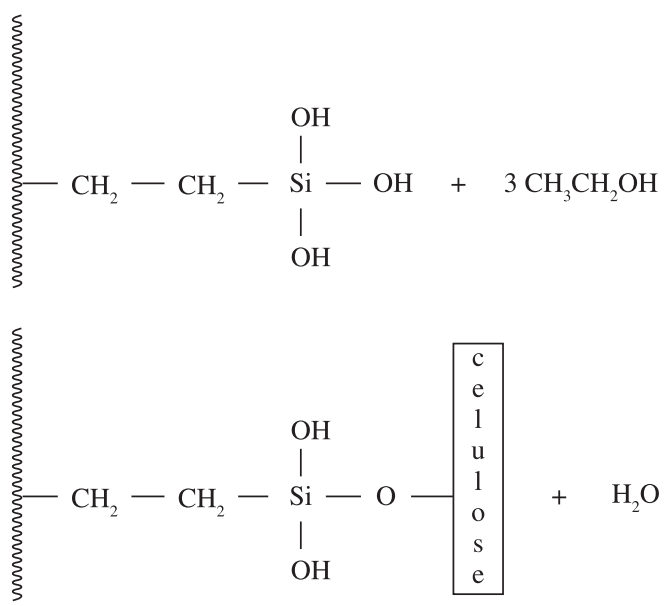


Tabela 1. Perfis de temperatura nos processos de extrusão.

\begin{tabular}{lcccc}
\hline & \multicolumn{4}{c}{ Temperatura das zonas de aquecimento $\left({ }^{\circ} \mathbf{C}\right)$} \\
\hline Condição 1 & 170 & 180 & 190 & 190 \\
Condição 2 & 170 & 190 & 200 & 190 \\
\hline
\end{tabular}

Tabela 2. Formulação dos compósitos.

\begin{tabular}{ccccc}
\hline Amostra & PP (\%) & FC (\%) & PPVTES (\%) & PPAM (\%) \\
\hline A & 100 & 0 & 0 & 0 \\
B & 70 & 30 & 0 & 0 \\
C & 68,5 & 30 & 1,5 & 0 \\
D & 68,5 & 30 & 0 & 1,5 \\
\hline
\end{tabular}

extrusora. Dois diferentes perfis de temperatura foram utilizados, conforme descritos na Tabela 1.

A composição dos sistemas processados é mostrada na Tabela 2. Foi utilizada uma razão de 0,05 entre as concentrações dos agentes de acoplamento e a concentração de fibras de coco, conforme os melhores resultados encontrados para compósitos similares preparados em câmara de mistura ${ }^{[20]}$.

Os pellets obtidos na extrusora foram moldados em uma injetora ROMI Primax R, utilizando o perfil de temperaturas de 210, 200, 190 e $180{ }^{\circ} \mathrm{C}$. Foram obtidos corpos de prova de acordo com a norma ASTM D-638-03 (corpos de prova tipo I). O molde possui um comprimento total de $170 \mathrm{~mm}$ e a área da seção transversal de $12 \times 4 \mathrm{~mm}^{2}$, tendo sido ajustado na temperatura de $75^{\circ} \mathrm{C}$.

\section{Caracterização}

Testes de tração foram realizados em Máquina Universal de Ensaios EMIC, modelo DL 10.000, velocidade de $5 \mathrm{~mm} / \mathrm{min}$, célula de carga de $5 \mathrm{kN}$, de acordo com a norma ASTM D-638-03. Fotomicrografias (MEV) foram obtidas com um Microscópio Eletrônico de Varredura JEOL, modelo JSM 6060. As superfícies das amostras fraturadas por tração receberam uma fina camada de ouro e foram analisadas com o equipamento operando com uma voltagem de aceleração de $10 \mathrm{kV}$. Para os testes de absorção de água, as amostras foram primeiramente secas a $60{ }^{\circ} \mathrm{C}$ por 8 horas antes da imersão em água destilada. Em determinados intervalos de tempo as amostras foram pesadas e o grau de absorção de água foi obtido utilizando a Equação 1:

$$
\text { Absorção de água }(\%)=\frac{\left(\mathrm{M}_{\mathrm{f}}-\mathrm{M}_{\mathrm{i}}\right)}{\mathrm{M}_{\mathrm{i}}} \times 100
$$

onde $\mathrm{M}_{\mathrm{f}}$ é a massa final após absorção de água e $\mathrm{M}_{\mathrm{i}}$ é a massa inicial da amostra seca. A quantidade de água absorvida foi obtida através da média de três corpos de prova de dimensões aproximadas de $30 \times 20 \times 3,5 \mathrm{~mm}$.

\section{Resultados e Discussão}

\section{Propriedades mecânicas}

De modo geral, a adição de cargas rígidas a matrizes poliméricas tende a produzir materiais mais rígidos e menos resistentes. A adição de fibras curtas de coco à matriz de PP mostrou esse tipo de comportamento, com aumento do módulo elástico (Figura 3). Esse aumento foi especialmente significativo nos materiais processados na temperatura mais alta (condição 2), onde foi verificado um aumento de $36 \%$ no módulo elástico do compósito não compatibilizado em comparação ao polímero puro. Considerando que o aumento na temperatura de processamento do PP puro levou a uma pequena redução no módulo elástico do material, duas razões

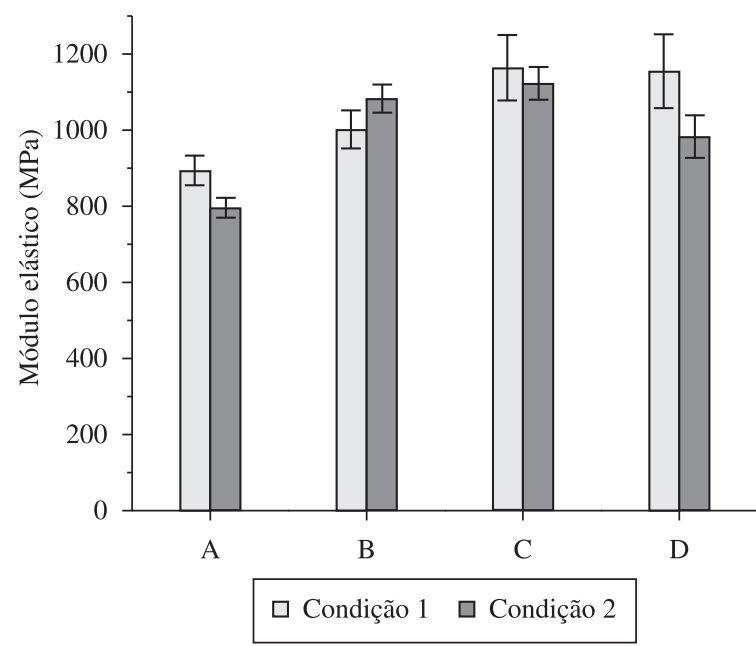

Figura 3. Módulo elástico do PP puro e dos compósitos preparados nas duas condições de processamento.

podem ser propostas para explicar o maior módulo dos compósitos na temperatura mais alta. Em primeiro lugar, a maior fluidez do polímero na temperatura de processamento diminui as forças de cisalhamento, minimizando a quebra das fibras de coco. Por outro lado, a maior degradação da matriz na temperatura mais alta facilita o alinhamento das fibras durante a injeção, aumentando a rigidez do material e seu módulo elástico.

Nos compósitos poliméricos o módulo elástico é geralmente influenciado pelo teor de carga, porém o efeito dos agentes de acoplamento sobre o módulo nem sempre é observado. Em alguns sistemas o grau de adesão interfacial entre os constituintes não tem mostrado efeito sobre o módulo ${ }^{[22,25]}$, enquanto que em outros aumentos têm sido observado ${ }^{[19,20]}$. Em compósitos de PP com fibras de coco preparados em câmara de mistura verificou-se um aumento de até $60 \%$ no módulo elástico pela adição de PPVTES e PPAM ${ }^{[20]}$. No presente trabalho, entretanto, o efeito dos mesmos agentes de acoplamento sobre o módulo não foi tão significativo. A adição de PPVTES e PPAM aos compósitos processados na condição de menor temperatura (condição 1) resultou em um pequeno acréscimo no módulo elástico, conforme mostrado na Figura 3, mostrando uma melhor transferência de esforços polímero-fibra no material compatibilizado. Entretanto, aumentando a temperatura de processamento não foi observado aumento significativo na propriedade, ao contrário, a adição de PPAM levou a uma redução do módulo elástico.

Os resultados dos testes de tração também mostraram que a adição de $30 \%$ de fibras de coco ao PP resulta em uma diminuição na resistência do material, com os compósitos suportando uma tensão máxima inferior ao PP puro, como pode ser visualizado na Figura 4.

A presença de fibras curtas dispersas produz descontinuidades na matriz polimérica, dificultando a distribuição e transferência do esforço aplicado, e assim, reduzindo a tensão máxima suportada pelos compósitos ${ }^{[26]}$. A baixa resistência verificada nos compósitos não compatibilizados também é resultante de falhas na interface, ocasionadas pela fraca interação dos constituintes. Os compósitos contendo $30 \%$ de fibras apresentaram tensão máxima inferior à do PP puro independentemente da condição de processamento utilizada. Analisando os resultados de tensão máxima suportada pelos compósitos contendo PPVTES e PPAM foi possível inferir que nesses sistemas a interação entre os constituintes melhorou e esse efeito foi maior nos compósitos preparados na condição 2 , 


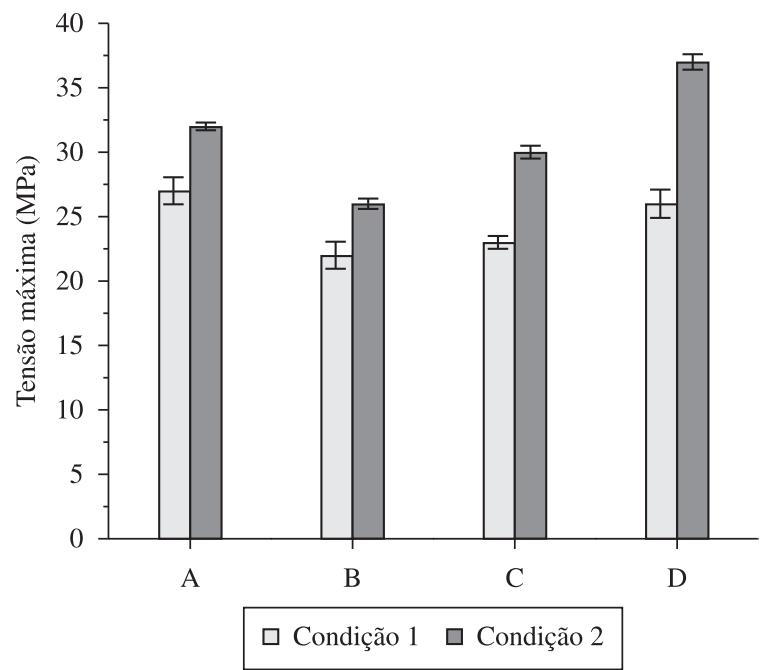

Figura 4. Tensão máxima do PP puro e dos compósitos preparados nas duas condições de processamento.

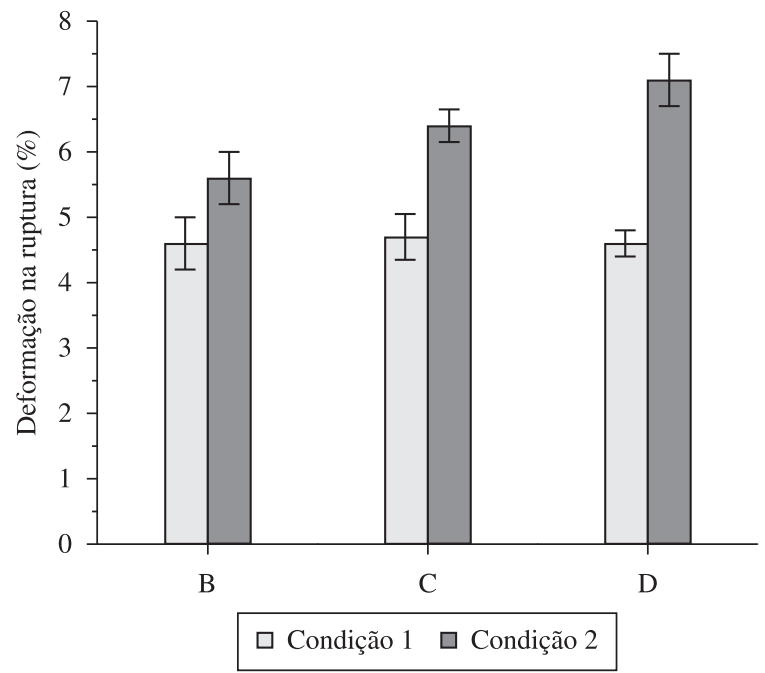

Figura 5. Deformação na ruptura dos compósitos nos testes de tração.

ou seja, o aumento no perfil de temperatura durante o processo de extrusão levou aos melhores resultados. Nessa condição a adição do PPVTES mostrou um aumento de $15 \%$ em comparação ao compósito não compatibilizado, porém o melhor resultado foi obtido para o compósito contendo PPAM, onde a tensão máxima ficou $42 \%$ acima da tensão máxima do compósito não compatibilizado, superior inclusive à tensão máxima do PP puro.

A Figura 5 mostra a deformação na ruptura sofrida pelos compósitos durante os testes de tração. O alongamento dos compósitos diminuiu em relação ao do PP puro processado, o qual não se rompeu nas condições em que os testes foram realizados. Foi possível verificar que a atuação do PPVTES e do PPAM, na condição 2, produziu um aumento na deformação, sendo que os compósitos que apresentaram os melhores resultados de tensão, também foram os que sofreram maior deformação.

\section{Morfologia}

Através da observação da superfície de fratura dos compósitos, utilizando microscopia eletrônica de varredura, é possível avaliar a dispersão das fibras na matriz bem como a atuação dos agentes de acoplamento na interface fibra/matriz. As micrografias das superfícies obtidas por fratura após testes de tração são mostradas na Figura 6. Para as duas condições de processamento, verificou-se a formação de superfícies pouco homogêneas e bastante deformadas nos compósitos sem agente de acoplamento (Figura 6a, b). Nesses sistemas, devido à fraca adesão interfacial, espaços vazios podem ser visualizados em torno das fibras, indicando a baixa molhabilidade da fibra pela matriz. O descolamento de fibras da matriz (pull-out) também é visível, indicando que neste caso o PP foi o principal responsável por suportar o esforço aplicado.

As superfícies dos compósitos contendo os agentes de acoplamento PPVTES e PPAM mostraram-se mais homogêneas que as dos compósitos não compatibilizados. Podem ser observadas fibras presas à matriz não deformada, sem espaços vazios na interface fibra/matriz, nos compósitos compatibilizados com PPVTES (Figura 6c, d), indicando melhor interação entre os componentes. Nos compósitos contendo PPAM observa-se que as fibras estão bastante aderidas à matriz de PP, sendo que na amostra preparada na condição 2 nem mesmo são observadas fibras salientes na superfície. Isso indica a existência de forte interação entre a fibra e a matriz, resultando em boa transferência da força de uma fase à outra. Nesse caso o rompimento da fibra durante o teste de tração mostra que o esforço é suportado tanto pela matriz quanto pela fase dispersa. Esse resultado justifica as melhores propriedades de tensão encontradas para os compósitos contendo PPAM e preparados na temperatura mais alta.

\section{Absorção de água}

O grau de absorção de água é uma importante característica a ser avaliada nos compósitos de fibras naturais, pois avalia o potencial desses materiais para utilização em certas aplicações, como por exemplo em aplicações externas nas quais exista contato com umidade. Dentre os efeitos adversos resultantes da absorção de água estão a perda das propriedades mecânicas, o favorecimento da biodegradabilidade e possíveis mudanças dimensionais que comprometem a performance do material a longo prazo $^{[27]}$. A absorção de água nos compósitos com fibras naturais ocorre devido à natureza higroscópica da fase dispersa, bem como através da difusividade por espaços vazios ou defeitos presentes ${ }^{[28]}$. A adição de agentes de acoplamento pode favorecer aspectos relacionados à absorção de umidade. Considerando que as moléculas de água são acumuladas nas fibras altamente hidrofílicas, espera-se que os agentes de acoplamento reduzam a absorção de água nesses sistemas ao interagirem com os grupos hidrofílicos da superfície das fibras e diminuírem os espaços vazios nas interfaces.

A Figura 7 mostra a evolução da absorção de água com relação ao tempo de imersão para os compósitos extrudados nas duas condições de processamento. Para todos os compósitos, a absorção de água aumentou com o tempo, não havendo estabilização da absorção até o final do período analisado (35 dias). O compósito não compatibilizado, preparado na condição de mais baixa temperatura (Amostra B1) apresentou a maior tendência à estabilização da absorção de água durante esse período. Comparando os sistemas não compatibilizados (Amostras B1 e B2) pode-se verificar que o compósito preparado na temperatura mais alta apresentou o maior grau de absorção de água. Isso pode ser o resultado de uma maior degradação dos materiais (fibras e matriz) quando submetidos a uma condição de aquecimento mais severo, com formação de produtos de oxidação, os quais têm maior afinidade com a água. $\mathrm{A}$ adição do PPVTES (Amostra $\mathrm{C} 1$ ) não alterou a resistência à absorção de umidade nos compósitos preparados com o perfil de temperatura de extrusão mais baixo, sendo que o PPAM (Amostra D1) aumentou um pouco essa absorção. Entretanto nos 


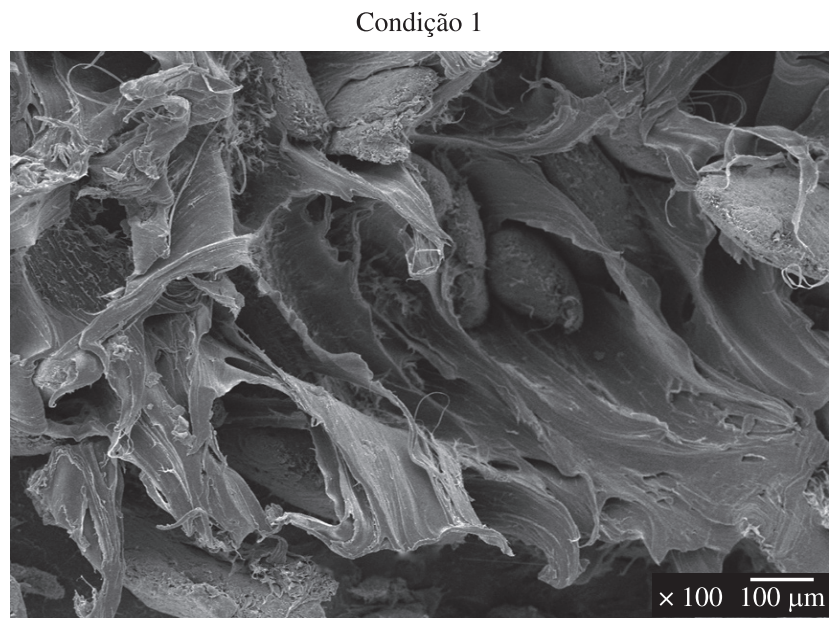

(a)

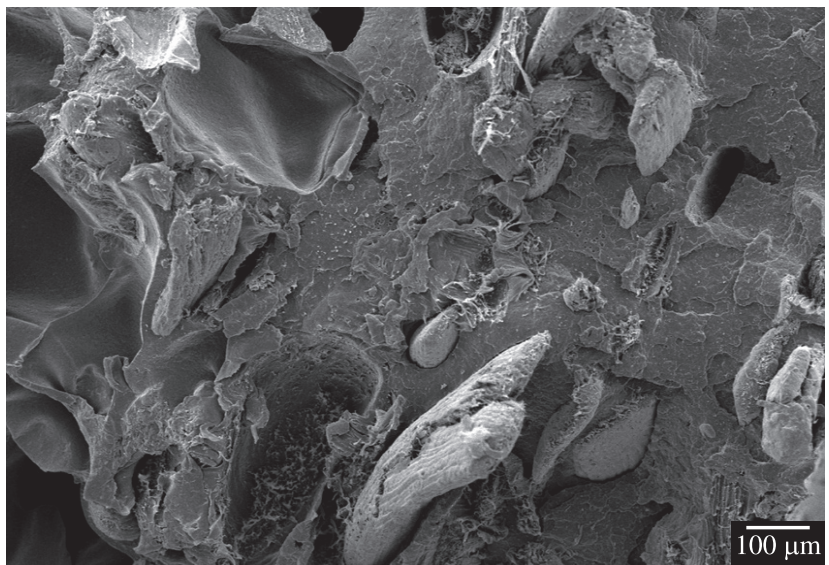

(c)

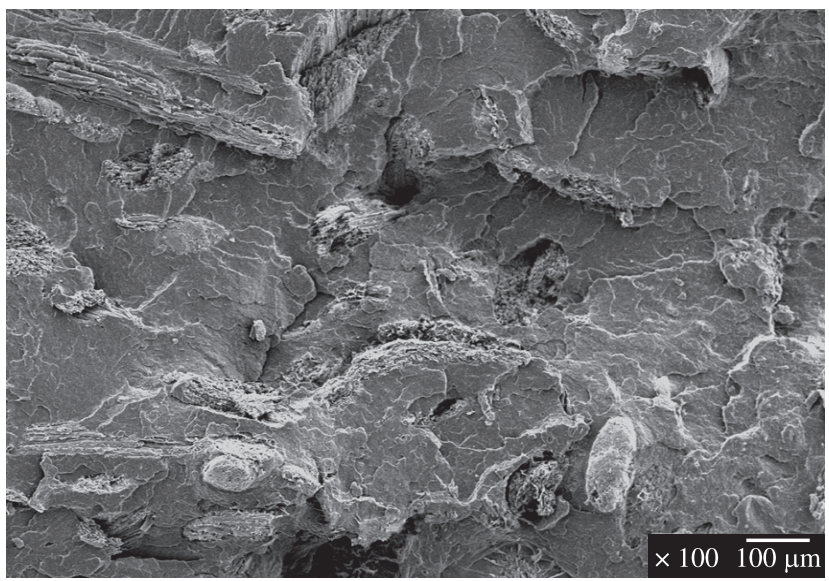

(e)
Condição 2

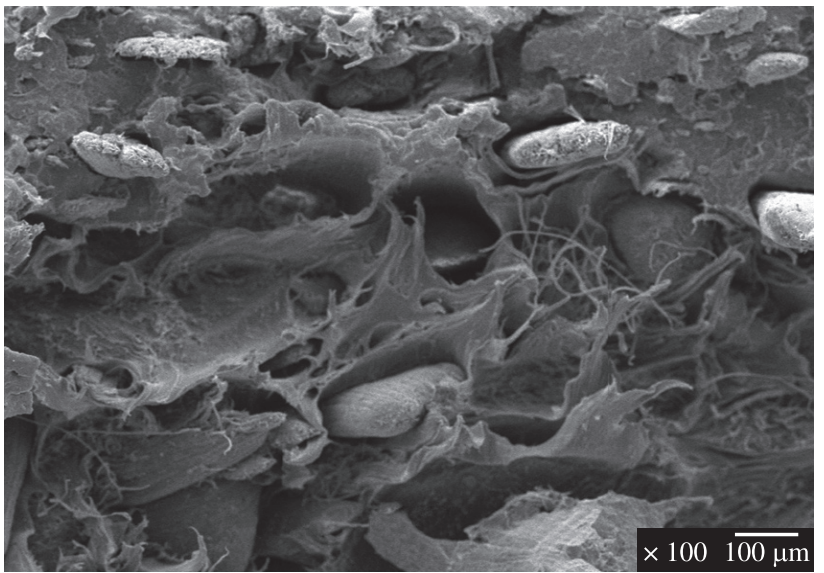

(b)

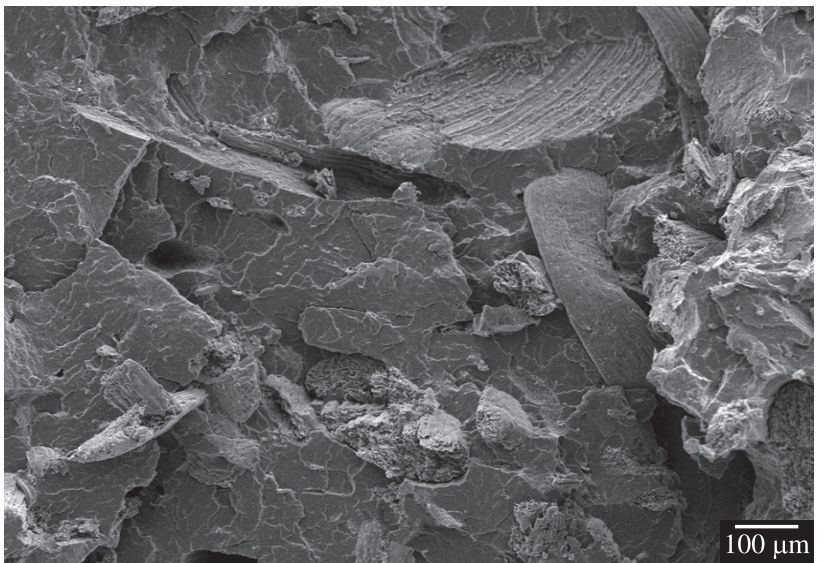

(d)

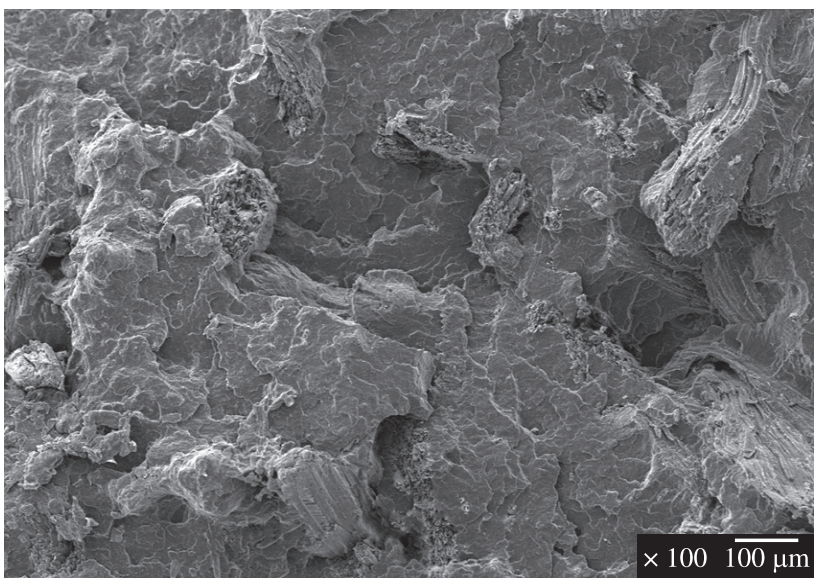

(f)

Figura 6. Micrografias dos compósitos contendo $30 \%$ de FC nas duas condições de extrusão: a) e b) sem agente de acoplamento; c) e d) com PPVTES; e) e f) com PPAM.

compósitos compatibilizados preparados com temperatura mais alta (Amostras B2 e C2) houve uma redução significativa no teor de água absorvida. Esse comportamento confirma o aumento da interação entre os componentes nos materiais processados na temperatura mais alta, diminuindo a disponibilidade dos sítios hidrofílicos das fibras. Assim pode-se concluir que a temperatura de processamento é uma variável que desempenha um papel muito importante na formação de ligações químicas durante o processo de compatibilização, especialmente em se tratando de processamento contínuo, onde o tempo de contato entre os componentes costuma ser curto.

Observou-se, ainda, que o agente de acoplamento à base de silano formou compósitos com menores graus de absorção de umidade que o agente de acoplamento à base de anidrido maleico. Isso pode ser explicado pela menor polaridade do grupo funcional alcoxisilano, que diminui o caráter hidrofílico do sistema. 


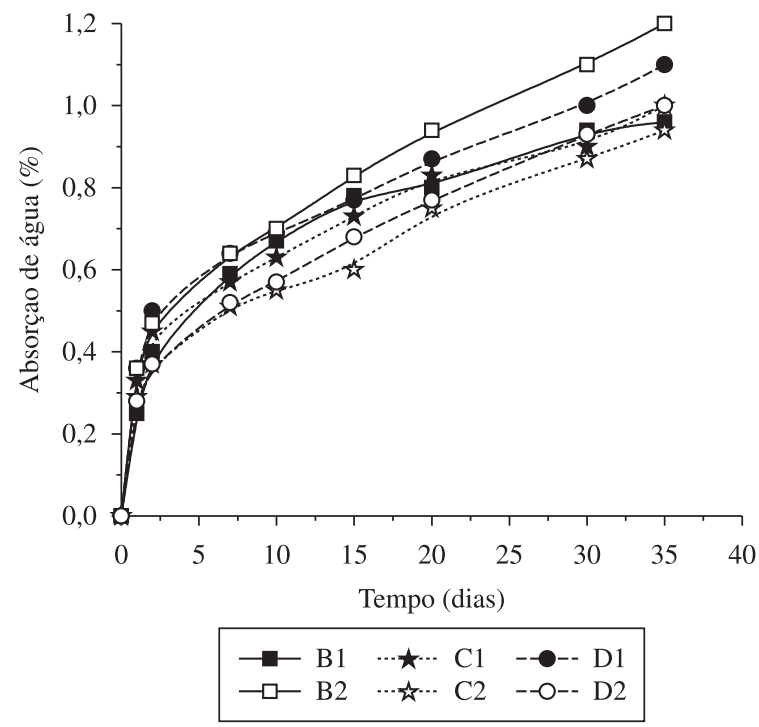

Figura 7. Quantidade de água absorvida versus tempo de imersão, nas duas condições de processamento: 1 (menor temperatura) e 2 (maior temperatura).

\section{Conclusões}

A utilização de fibras curtas de coco, um resíduo de baixo custo, na preparação de compósitos com PP, em diferentes perfis de extrusão, produziu compósitos com maior módulo, porém com menor resistência à tração, comparativamente à matriz pura processada nas mesmas condições. A adição de PP modificado com vinilalcoxisilano e com anidrido maleico aumentou a resistência mecânica dos materiais. Os resultados mostraram que as propriedades dos materiais apresentam forte dependência com o perfil de temperatura de processamento utilizado. $\mathrm{O}$ aumento de temperatura durante o processo de extrusão, na presença dos agentes de acoplamento, resultou nos compósitos com as melhores propriedades mecânicas. Esse comportamento está relacionado ao estabelecimento de novas ligações químicas entre o polímero funcionalizado e a superfície das fibras de coco, aumentando a interação entre os componentes dos sistemas.

Embora o maior módulo elástico tenha sido obtido nos compósitos compatibilizados com PP silanizado, a maior resistência à tração foi obtida nos compósitos contendo PP modificado com anidrido maleico. Esses compósitos também apresentaram superfícies mais homogêneas quando analisados por microscopia eletrônica de varredura após os testes de tração, indicando maior adesão entre a fibra e a matriz, especialmente nos compósitos preparados sob mais alta temperatura de processamento.

Nos compósitos não compatibilizados observou-se que a resistência à absorção de água diminuiu com o aumento na temperatura de processamento, provavelmente devido ao aumento de reações de oxidação, com formação de novos grupos hidrofílicos nos materiais. Entretanto, na presença dos agentes de acoplamento esse comportamento se inverteu. Os menores graus de absorção de água foram obtidos para os compósitos preparados com PP modificado com silano, preparados com o perfil de temperatura mais elevado. Esse resultado pode ser atribuído ao comprometimento dos grupos hidrofílicos pela interação com o agente de acoplamento, mais acentuado nos compósitos preparados em temperatura mais elevada.

\section{Agradecimentos}

Os autores agradecem à BRASKEM e AMAFIBRA pelo fornecimento dos materiais e à UFRGS e CAPES pelo suporte financeiro a este trabalho.

\section{Referências Bibliográficas}

1. Baillie, C. -“Green Composites”, Woodhead Publishing, Cambridge (2004).

2. Marinelli, A. L.; Monteiro, M. R.; Ambrósio, J. D.; Branciforti, M. C.; Kobayashi, M. \& Nobre, A. D. - Polímeros, 18, p.92 (2008).

3. Ashori, A. - Biores. Techn., 99, p.4661 (2008).

4. Wielage, B.; Lampke, T.; Utschik, H \& Soergel, F. - J. Mater. Proces. Technol., 139, p.140 (2003).

5. Gomes, A.; Matsuo, T.; Goda, K. \& Ohgi, J. - Compos. Part A: Appl. Sci. Manufac., 38, p.1811 (2007).

6. Satyanarayana, K. G.; Guimarães, J. L.\& Wypych, W. - Compos. Part A: Appl. Sci. Manufac., 38, p.1694 (2007).

7. Ishizaki, M. H.; Visconte, L. Y.; Furtado, R. G.; Leite, M. C. A. M. \& Leblanc, J. L. - Polímeros, 16, p.182 (2006).

8. Schuh, T. G. \& Gayer, U. - "Automotive applications of natural fiber composites", in: Lignocellulosic-Plastic Composites, A. L. Leão, F. X. Carvalho \& E. Frollini (eds.), UNESP Publishers, Botucatu (1997).

9. Li, X.; Tabil, L. G. \& Panigrahi, S. - J. Polym. Environ.,15, p.25 (2007).

10. Correa, C. A.; Fonseca, C. N. P.: Neves, S.; Razzino, C. A. \& Hage Jr., E. Polímeros, 13, p.154 (2003).

11. Rosa, S. M. L.; Ferreira, C. A. \& Nachtigall, S. M. B. - Macromol. Res.,17, p.8 (2009).

12. Kim, H. S.; Lee, B. H.; Choi, S. W.; Kim, S. \& Kim, H. - J. Compos. Part A: Appl. Sci. Manufac., p.1473 (2007).

13. Doan, T. T. L.; Gao, S. L. \& Mäder, E. - Compos. Sci. Technol., 66, p.952 (2006).

14. Plueddemann, E. P. “Silane Coupling Agents”, Plenum, New York (1991).

15. Li, Y.; Mai, Y. W. \& Ye, L. - Compos. Sci. and Technol., 60, p.2037 (2000).

16. Bel-Hassen, R.; Boufi, S.; Salon, M. C. B.; Abdelmouleh, M. \& Belgacem, M. N. - J. of Appl. Polym. Sci., 108, p.1958 (2008).

17. Arbelaiz, A.; Fernández, B.; Cantero, G.; Llano-Ponte, R.; Valea, A. \& Mondragon, I. - J. Compos. Part A: Appl. Sci. Manufac., 36, p.1637 (2005).

18. Fabris, F. W.; Cardozo, N. S. M.; Mauler, R. S. \& Nachtigall, S. M. B. Polym. Compos., 30, p.872 (2009).

19. Nachtigall, S. M. B.; Cerveira, G. S. \& Rosa, S. M. L. - Polym. Test., 26, p.619 (2007).

20. Santos, E. F.; Mauler, R. S. \& Nachtigall, S. M. B. - J. Reinf. Plast. Compos., 28, p.2119 (2009).

21. Bengston, M.; Baillif, L. M. \& Oskman, K. - Compos. Part A: Appl. Sci. Manufact., 38, p.1922 (2007).

22. Doan, T. T. L.; Gao, S. L. \& Mäder, E. - Comp. Sci. Tech., 66, p.952 (2006).

23. Quijano-Solis, C.; Yan, N. \& Zhang, S.Y. - J. Compos. Part A: Appl. Sci. Manufac., 40, p.351(2009).

24. Fabris, F. W.; Stedile, F. C.; Mauler, R. S. \& Nachtigall, S. M.B. - Eur. Polym. J., 40, p.1119 (2004).

25. Pracella, M.; Chionna, D.; Anguillesi, I.; Kulinski, Z. \& Piorkowska, E. - Comp. Sci. Tech., 66, p.2218 (2006).

26. Yang, H. S.; Wolcott, M. P.; Kim, H. S.; Kim, S. \& Kim, H. J. - Polym. Test., 25, p.668 (2006).

27. Sharma, S. C.; Krishna, M.; Narasimhamurthy, H. N. \& Sanjeevamurth J. Reinf. Plast. Compos., 25, p.925 (2006).

28. Wang, W.; Sain, M. \& Cooper, P. A. - Compos. Sci. Tech., 66, p.379 (2006).

Enviado: 30/09/09

Reenviado: 10/03/10

Aceito: $24 / 03 / 10$

DOI: $10.1590 / \mathrm{S} 0104-14282010005000036$ 